\title{
Spatial stability in linear thermoelasticity
}

\author{
R. J. Knops \\ The Maxwell Institute of Mathematical Sciences \\ Heriot-Watt University, \\ Edinburgh, Scotland \\ R. Quintanilla \\ Matemática Aplicada 2,UPC \\ C.Colón 11, 08222 Terrassa, \\ Barcelona, Spain
}

November 12, 2013

\begin{abstract}
Uniqueness and spatial stability are investigated for smooth solutions to boundary value problems in non-classical linearised and linear thermoelasticity subject to certain conditions on material coefficients. Uniqueness is derived for standard boundary conditions on bounded regions using a generalisation of Kirchhoff's method. Spatial stability is discussed for the semi-infinite prismatic cylinder in the absence of specified axial asymptotic behaviour. Alternative growth and decay estimates are established principally for the cross-sectional energy flux that is shown to satisfy a first order differential inequality. Uniqueness in the class of solutions with bounded energy follows as a corollary.

Separate discussion is required for the linearised and linear theories. Although the general approach is similar for both theories, the argument must be considerably modified for the treatment of the linear theory.
\end{abstract}




\title{
Spatial stability in linear thermoelasticity
}

\author{
November 12, 2013
}

\begin{abstract}
Uniqueness and spatial stability are investigated for smooth solutions to boundary value problems in non-classical linearised and linear thermoelasticity subject to certain conditions on material coefficients. Uniqueness is derived for standard boundary conditions on bounded regions using a generalisation of Kirchhoff's method. Spatial stability is discussed for the semi-infinite prismatic cylinder in the absence of specified axial asymptotic behaviour. Alternative growth and decay estimates are established principally for the cross-sectional energy flux that is shown to satisfy a first order differential inequality. Uniqueness in the class of solutions with bounded energy follows as a corollary.

Separate discussion is required for the linearised and linear theories. Although the general approach is similar for both theories, the argument must be considerably modified for the treatment of the linear theory.
\end{abstract}

\section{Introduction}

Spatial stability is a particular, but important, type of continuous data dependence. For boundary value problems on a bounded or unbounded region, the concept refers to the spatial behaviour of the solution with respect to distance from that part of the boundary on which non-zero data is specified. In most studies, behaviour cannot be measured pointwise, but instead must be analysed in some average sense, such as a cross-sectional $L_{2}$ norm. Uniqueness of the solution in the class of bounded energy usually is an easy consequence.

Various techniques for spatial stability, fully reviewed in $[9,7,8]$, have been developed in the literature, originally for linear isothermal isotropic elasticity. Many examine behaviour on cylindrical regions and adopt a volume energy function as a measure of the solution. By contrast, the approach employed in the present study extends the procedure first developed in [4], and measures the solution by means of the cross-sectional energy flux. For simplicity, attention is confined to three-dimensional prismatic cylindrical regions, but our treatment explicitly includes nonhomogeneous anisotropic linearised and linear thermoelastostatic theories. The original investigation undertaken in [4] establishes alternative growth or decay behaviour, the respective rates indicating how far edge effects penetrate into the region. This feature is preserved in the present 
discussion for both the linearised and classical linear theories of thermoelastostatics.

In order for the paper to be reasonably self-contained, there is inevitable overlap with other publications. In particular, appropriate sections of the concise survey [15], which deals solely with the linearised theory, are reproduced and amplified not only for ease of reference, but also to facilitate comparison with the new proofs and results in the linear theory obtained in the paper.

Notation, introduced in Section 2, is either direct or indicial according to the particular context. A subscript comma denotes partial differentiation, while repeated Latin suffixes indicate summation over the range 1,2,3. Repeated Greek subscripts denote summation over 1,2 . Section 2 also states the boundary value problems to be considered in both the linearised and linear theories, explains their interrelation, and postulates certain sign-definite assumptions sufficient for the validity of subequent conclusions. Uniqueness of smooth solutions to standard boundary value problems in the linearised and linear theories is established for bounded regions in Section 3. Section 4 concerns non-classical linearised thermoelastostatics, and treats a non-homogeneous anisotropic material that occupies a semi-infinite cylinder, supposed prismatic for simplicity, in equilibrium under zero source terms (body force and heat supply), and subject to homogeneous lateral boundary data. The derivation and integration of a differential inequality to obtain respectively an exponentially increasing lower bound and an exponentially decreasing upper bound for alternative growth and decay of the cross-sectional energy flux expands the discussion presented in [15]. The decay estimate is employed to demonstrate uniqueness of the solution to the prescribed boundary value problem in the class of bounded total energy. Discussion of the linear theory, not presented previously in the literature and undertaken in Section 5, is generally similar to that for Section 4, but differs in important detail and requires significant modication of the proofs. The main difficulty occurs when the displacement gradient is necessarily replaced by the linear strain. Consequently, the quadratic form corresponding to the linear strain energy function is restricted to be positive-definite on the set of symmetric second order tensors. This in turn requires the introduction of a generalised Korn's inequality for the construction of the differential inequality governing the spatial evolution of the cross-sectional energy flux. The subsequent discussion proceeds as for the linearised theory, apart from the estimate of the amplitude in terms of the base data, which again involves important modification to the previous argument. Uniqueness in the class of bounded energy is implied by the decay estimate. A final section contains brief comment on spatial stability for non-cylindrical unbounded regions, and speculates on the general occurrence of algebraic decay rates. The interested reader may consult [15] for elaboration of these remarks.

Throughout, a solution is assumed to exist that is of sufficient smoothness to justify the calculations. 


\section{Notation and other preliminaries}

Elements of linearised and linear thermoelastostatics relevant to the present study are presented in Sections 2.1 and 2.2, respectively. For either theory, the material in its reference configuration occupies a bounded or unbounded region $\Omega$ of $\mathbb{R}^{3}$ whose boundary $\partial \Omega$ is supposed sufficiently smooth to admit application of the divergence theorem. The same rectangular coordinate system is used in the discussion of both theories.

\subsection{Linearised thermoelastostatics}

The deformation and thermal terms in the so-called linearised theory are derived as small perturbations of corresponding large quantities in the primary full nonlinear theory, and is otherwise known as the theory of small deformations superposed upon large deformations.

Let a point at $X_{i}$ in the reference configuration $\Omega$ be deformed into points $x_{i}^{(1)}$ in the primary configuration and $x_{i}^{(2)}$ in the secondary configuration. The equilibrium equations for the increment between the large primary and secondary deformations whose respective components of the first Piola-Kirchhoff stress tensors are $t_{i j}^{(1)}, t_{i j}^{(2)}$ and of the heat fluxes are $q_{i}^{(1)}, q_{i}^{(2)}$, have the form

$$
\begin{aligned}
t_{i j, j}+\rho_{0} f_{i} & =0, & & x \in \Omega, \\
q_{i, i}+\rho_{0} s & =0, & & x \in \Omega,
\end{aligned}
$$

where differentiation is with respect to $x_{i}^{(1)}, \rho_{0}$ is the mass density of the body occupying $\Omega$, and $t_{i j}=t_{i j}^{(2)}-t_{i j}^{(1)}, q_{i}=q_{i}^{(2)}-q_{i}^{(1)}$. Furthermore, $f_{i}=f_{i}^{(2)}-f_{i}^{(1)}$ are components of the increment in the body force per unit mass, and $s=$ $s^{(2)}-s^{(1)}$ is the increment in the scalar heat supply per unit mass. Superscripts 1,2 indicate that quantities belong to either the large primary or secondary states. The corresponding increments in the vector displacement $u_{i}=u_{i}^{(2)}-$ $u_{i}^{(1)}$ and scalar temperature $\theta=\theta^{(2)}-\theta^{(1)}$ are supposed to be continuously differentiable. It is also supposed that $t_{i j}$ and $q_{i}$ can be expanded in series about the primary state and that terms of order higher than the first can be neglected. There is no loss in confusing spatial differentiation with respect to the primary configuration and that with respect to the secondary configuration to the order implied by linearisation. Accordingly, in what follows we set $x_{i}=x_{i}^{(1)}=x_{i}^{(2)}$ in linearised theories. These operations lead to coupled constitutive relations for the linearised increments of stress and heat flux given by (eg., [6])

$$
\begin{aligned}
t_{i j} & =d_{i j k l} u_{k, l}+\beta_{i j} \theta, \quad x \in \Omega, \\
q_{i} & =h_{i j k} u_{j, k}+a_{i} \theta+k_{i j} \theta_{, j}, \quad x \in \Omega,
\end{aligned}
$$

where $\beta_{i j}(x)$ is the non-symmetric thermal coupling tensor, $k_{i j}(x)$ is the heat conduction tensor, $h_{i j k}(x)$ and $a_{i}(x)$ are other constitutive incremental tensors, and $d_{i j k l}(x)$ is the incremental elasticity tensor that possesses only the major 
symmetry

$$
d_{i j k l}=d_{k l i j} .
$$

The linear elasticities $c_{i j k l}(x)$ of the unstressed reference configuration possess both the major and minor symmetries

$$
c_{i j k l}=c_{k l i j}=c_{j i k l},
$$

and are related to the incremental elasticities by

$$
d_{i j k l}=c_{i j k l}+\sigma_{i k} \delta_{j l},
$$

where $\sigma_{i j}$ is the symmetric Cauchy stress in the deformed primary configuration, and $\delta_{i j}$ is the usual Kronecker delta function.

Substitution of the constitutive relations (2.3) and (2.4) in the equilibrium equations (2.1) and (2.2) yields

$$
\begin{aligned}
\left(d_{i j k l} u_{k, l}+\beta_{i j} \theta\right)_{, j}+\rho_{0} f_{i} & =0, & x \in \Omega, \\
\left(h_{i j k} u_{j, k}+a_{i} \theta+k_{i l} \theta_{, l}\right)_{, i}+\rho_{0} s & =0, & x \in \Omega,
\end{aligned}
$$

to which must be adjoined prescribed kinematical and thermal boundary conditions.

Assume that $\Omega$ is bounded with smooth boundary $\partial \Omega$. For convenience, the boundary conditions mainly selected for study are those of Dirichlet:

$$
u_{i}(x)=\bar{u}_{i}(x), \quad \theta(x)=\bar{\theta}(x), \quad x \in \partial \Omega,
$$

where $\bar{u}_{i}(x)$ and $\bar{\theta}(x)$ are prescribed functions.

In general, the kinematical and thermal constitutive coefficients are differentiable functions of position $x_{i}$ through dependence upon the primary configuration, or upon an inhomogeneous reference state. The same dependence implies that the coefficients $d_{i j k l}$ become sign-definite only for certain primary configurations. Aspects of this topic for nonlinear elasticity are discussed in [16, Sections 53, 56].

When the temperature is uniform in the primary configuration $\left(\theta_{i}^{(1)}=0\right)$, but the primary stress is non-zero, the incremental heat flux vector simplifies to

$$
q_{i}=k_{i j} \theta_{, j}, \quad x \in \Omega
$$

and

$$
h_{i j k}=0, \quad a_{i}=0,
$$

so that the heat conduction equation (2.7) reduces to

$$
\left(k_{i j} \theta_{, i}\right)_{, j}+\rho_{0} s=0 .
$$

With special reference to the linearised theory, we suppose that:

(I) The thermal conductivity tensor is positive-definite in the sense that there exists a specified positive constant $k_{1}$ such that

$$
k_{1} \zeta_{i} \zeta_{i} \leq k_{i j} \zeta_{i} \zeta_{j}
$$


It is immediately obvious that for the purposes of this assumption, there is no loss in supposing that $k_{i j}$ is a symmetric tensor.

(II) There exists a given positive constant $d_{1}$ such that

$$
d_{1} \xi_{i j} \xi_{i j} \leq d_{i j k l} \xi_{i j} \xi_{k l}, \quad \forall \xi_{i j}
$$

We also impose two supplementary conditions, the first of which is

$$
A \equiv k_{1}-\left(\frac{\widetilde{a}^{2}}{\lambda_{1}(\Omega)}\right)^{1 / 2}>0
$$

where

$$
\widetilde{a}^{2}=\max _{\Omega} a_{i} a_{i}
$$

and $\lambda_{1}(\Omega)$ is the first eigenvalue for the fixed membrane problem for $\Omega$, so that

$$
\lambda_{1}(\Omega) \int_{\Omega} \theta^{2} d x \leq \int_{\Omega} \theta_{, i} \theta_{, i} d x .
$$

The second supplementary condition supposes that the coefficients $\beta_{i j}, h_{i j k}$, $a_{i}, d_{1}, k_{1}$ satisfy

$$
F \equiv \lambda_{1}(\Omega) d_{1}^{2} A^{2}-\widetilde{\beta}^{2} \widetilde{h}^{2}>0
$$

where

$$
\begin{aligned}
\widetilde{\beta}^{2} & =\max _{\Omega} \beta_{i j} \beta_{i j}, \\
\widetilde{h}^{2} & =\max _{\Omega} h_{i j k} h_{i j k} .
\end{aligned}
$$

A necessary and sufficient condition for assumption (2.17) is

$$
\lambda_{1}^{1 / 2}(\Omega) d_{1} A-\widetilde{\beta} \widetilde{h}>0 .
$$

Assumptions (I) and (II) are consistent with physical experience. Inequality (2.12) states that the heat conductor tensor is positive-definite, which strengthens the non-negative condition derived from thermodynamics. Inequality (2.13), within the context of elastic stability theory, is sufficient for the dynamic stability of the null solution in linearised elastodynamics (cp., [16]). The supplementary conditions guarantee the sign-definiteness of a certain bilinear quadratic form vital in subsequent developments. Note that (2.17) holds trivially when the temperature in the primary configuration is uniform by virtue of $(2.10)$.

Sign-definiteness in linear elastic theories cannot be deduced from thermodynamics with the exception, as just remarked, of the heat conduction tensor which is non-negative. Even arguments based upon stability, precisely or colloquially defined, are unreliable since often they are tautological. Moreover, in linearised theories, several coefficients depend crucially upon the primary state and therefore may become indefinite indicating, for example, the onset of bifurcation. Consequently, sign-definiteness must be introduced as a separate postulate. 


\section{$2.2 \quad$ Linear thermoelastostatics}

The equilibrium equations for the linear theory may be derived from those for the linearised theory when the primary configuration has uniform temperature, zero stress, and null deformation so that $\theta^{(1)}$ is constant, and $u_{i}^{(1)}=\sigma_{i j}=0$. The reference, primary and secondary states may be confused, and in the notation of Section 2.1 we may set $x_{i}=x_{i}^{(1)}=x_{i}^{(2)}=X_{i}$. Under these conditions, we have $d_{i j k l}=c_{i j k l}$, while the constitutive relations (2.3) and (2.4) become

$$
\begin{aligned}
t_{i j} & =c_{i j k l} e_{k l}+\beta_{i j} \theta, \quad x \in \Omega, \\
q_{i} & =k_{i j} \theta_{, j}, \quad x \in \Omega,
\end{aligned}
$$

where

$$
e_{i j}=\frac{1}{2}\left(u_{i, j}+u_{j, i}\right)
$$

on noting the minor symmetry $(2.5)_{2}$. In the linear theory, the stress is symmetric, so that $t_{i j}=t_{j i}$, and the symmetries (2.5) together with (2.21) imply

$$
\beta_{i j}=\beta_{j i}
$$

Substitution of the constitutive relations (2.21) in the equilibrium equations (2.1) leads to

$$
\left(c_{i j k l} e_{k l}\right)_{, j}+\left(\beta_{i j} \theta\right)_{, j}+\rho_{0} f_{i}=0, \quad x \in \Omega .
$$

The thermal coefficients vanish in accordance with (2.10) and by (2.22), the heat conduction equation (2.2) reduces to the simplified form (2.11).

Boundary conditions necessary to complete the specification of the boundary value problem (2.25) and (2.11) are the same as those previously mentioned.

We retain the positive-definite assumption (2.12) for the heat conduction tensor, but replace condition (2.13) by the stricter requirement that the linear elasticities are positive-definite on the set of symmetric tensors, so that

$$
c_{0} \psi_{i j} \psi_{i j} \leq c_{i j k l} \psi_{i j} \psi_{k l}, \quad \forall \psi_{i j}=\psi_{j i}
$$

where $c_{0}$ is a given positive constant.

It will emerge that this condition demands significant modification of the proof of spatial stability, and to a lesser extent, of uniqueness. These topics are discussed in the next section.

\section{Uniqueness on a bounded region}

The systems of linear equations introduced in the previous section hold pointwise in $\Omega$ and consequently the solutions must be continuously differentiable to appropriate order. Otherwise the equations cannot be satisfied at all points of $\Omega$. Such solutions are called strong or classical solutions in contrast to weak solutions which possess less differentiability and satisfy the equations only in a 
weak or averaged (i.e., integral) sense. Our concern here is solely with strong solutions which throughout are assumed to exist.

We seek conditions on the constitutive functions that ensure that the boundary value problems stated in Sections 2.1 and 2.2 possess a unique strong solution on bounded regions $\Omega$. Uniqueness in unbounded regions is considered in subsequent sections. The conditions, which depend upon the type of boundary value problem and are imposed on the elastic moduli and elasticities respectively, usually are sufficient but not necessary, and generalise those employed in Kirchhoff's classical proof. We emphasise, however, that uniqueness may hold under conditions other than sign-definiteness.

As illustration of the technique, we discuss uniqueness of a strong solution to the Dirichlet boundary value problem. By linearity, uniqueness is equivalent to proving that at most the null solution exists subject to homogeneous boundary data and source terms. That is, for the purposes of the proof, we assume that $\tilde{u}_{i}=\tilde{\theta}=f_{i}=s=0$.

\subsection{Uniqueness in linearised thermoelastostatics}

In this section, we prove the following theorem:

Theorem 3.1 . Let us to assume that conditions (I) and (II) are satisfied and that the inequalities (2.14) and (2.17) hold. Then the boundary value problem (2.6) and (2.7) for presribed source terms and subject to (2.8) possesses a unique solution.

Proof: For prescribed boundary data, body-force, and heat supply, consider two different solutions to the boundary value problem (2.6) and (2.7) and let $u_{i}$ and $\theta$ denote the difference of the displacement and temperature between the two solutions respectively. Consequently, with this notation, we may set $f_{i}=s=0$, and then multiply $(2.6)$ by $u_{i}$, add to (2.7) multiplied by $\Gamma \theta$, where $\Gamma$ is a positive constant to be chosen, and on integrate by parts over $\Omega$, to obtain

$$
\int_{\Omega} B\left(u_{i, j}, \theta_{, i}, \theta\right) d x=0
$$

where

$$
B\left(u_{i, j}, \theta_{, i}, \theta\right)=d_{i j k l} u_{i, j} u_{k, l}+\beta_{i j} u_{i, j} \theta+\Gamma h_{i j k} u_{j, k} \theta_{, i}+\Gamma a_{i} \theta_{, i} \theta+\Gamma k_{i j} \theta_{, i} \theta_{, j} .
$$

An application of the Schwarz, Poincaré, and arithmetic-geometric mean inequalities yields the inequalities

$$
\begin{aligned}
\pm \int_{\Omega} \beta_{i j} u_{i, j} \theta d x & \leq \frac{\widetilde{\beta}^{2} \alpha_{1}}{2} \int_{\Omega} u_{i, j} u_{i, j} d x+\frac{1}{2 \alpha_{1} \lambda_{1}(\Omega)} \int_{\Omega} \theta_{, i} \theta_{, i} d x \\
\pm \int_{\Omega} h_{i j k} u_{j, k} \theta_{, i} d x & \leq \frac{\alpha_{2} \widetilde{h}^{2}}{2} \int_{\Omega} u_{i, j} u_{i, j} d x+\frac{1}{2 \alpha_{2}} \int_{\Omega} \theta_{, i} \theta_{, i} d x \\
\pm \int_{\Omega} a_{i} \theta \theta_{, i} d x & \leq \frac{1}{2}\left(\widetilde{a}^{2} \alpha_{3}+\frac{1}{\alpha_{3} \lambda_{1}(\Omega)}\right) \int_{\Omega} \theta_{, i} \theta_{, i} d x
\end{aligned}
$$


where $\alpha_{1}, \alpha_{2}, \alpha_{3}$ are arbitrary positive constants, and $\widetilde{a}, \widetilde{\beta}$ and $\widetilde{h}$ are defined by $(2.15),(2.18)$, and (2.19) respectively.

After an appeal to assumptions (2.12) and (2.13), we have

$$
\int_{\Omega} B d x \geq \int_{\Omega}\left(c_{1} u_{i, j} u_{i, j}+c_{2} \theta_{, i} \theta_{, i}\right) d x
$$

in which the constants $c_{1}, c_{2}$ are given by

$$
\begin{aligned}
& c_{1}=d_{1}-\frac{\alpha_{1} \widetilde{\beta}^{2}}{2}-\frac{\Gamma \alpha_{2} \widetilde{h}^{2}}{2} \\
& c_{2}=\left(\Gamma k_{1}-\frac{1}{2 \alpha_{1} \lambda_{1}(\Omega)}-\frac{\Gamma}{2 \alpha_{2}}-\frac{\Gamma}{2}\left(\widetilde{a}^{2} \alpha_{3}+\frac{1}{\alpha_{3} \lambda_{1}(\Omega)}\right)\right) .
\end{aligned}
$$

Let us set

$$
\begin{aligned}
\alpha_{1} \widetilde{\beta}^{2} & =\alpha_{2} \Gamma \widetilde{h}^{2}=\frac{\left(\widetilde{\beta}^{2} \widetilde{h}^{2}+3 \lambda_{1}(\Omega) d_{1}^{2} A^{2}\right)}{4 \lambda_{1}(\Omega) d_{1} A^{2}} \\
\alpha_{3} & =\left(\lambda_{1}(\Omega) \widetilde{a}^{2}\right)^{-1 / 2} \\
\Gamma & =d_{1} A \widetilde{h}^{-2}
\end{aligned}
$$

to recover

$$
\begin{aligned}
c_{1} & =\frac{F}{4 \lambda_{1}(\Omega) d_{1} A^{2}}>0, \\
c_{2} & =F \frac{d_{1} A^{2}}{\widetilde{h}^{2}\left(\widetilde{\beta}^{2} \widetilde{h}^{2}+3 \lambda_{1}(\Omega) d_{1}^{2} A^{2}\right)}>0,
\end{aligned}
$$

where $A(>0)$ and $F(>0)$ are defined respectively by (2.14) and (2.17). We finally conclude from (3.6) that

$$
\int_{\Omega} B d x \geq 0
$$

with equality if and only if $u_{i}, \theta$ are identically zero in $\Omega$. The condition (3.7) along with (3.1) implies that $u_{i}$ and $\theta$ must vanish. Thus, only the trivial solution exists and uniqueness is established.

Note, in particular, that (2.12) and (2.13) are sufficient but not necessary conditions. Uniqueness also may hold under different sets of sufficient conditions; for example, when the quadratic form (3.2) is negative-definite, which may occur for certain primary stressed configurations.

As mentioned previously, when the temperature is uniform in the primary configuration, the governing equations reduce to the pair (2.6) and (2.11) and therefore become uncoupled. A strong solution to the heat conduction equation (2.11) exists and is unique for Dirichlet data provided the heat conduction tensor satisfies the positive-definite condition (2.12). The temperature therefore may be assumed known and consequently supplements the source terms in (2.6). A 
strong solution exists to the latter equation subject to Dirichlet data and the positive-definite condition (2.13) by the theory of elliptic equations. Moreover, homogeneous thermal data implies $\theta(x) \equiv 0$, and conditions on the coefficients $d_{i j k l}$ for uniqueness depend solely upon those for the homogeneous equation

$$
\left(d_{i j k l} u_{k, l}\right)_{, j}=0, \quad x \in \Omega
$$

studied in [11].

Similar comments apply to the mixed and Neumann boundary value problems, although it must be observed that for the Neumann boundary value problem, the temperatue is unique only to within an arbitrary additive constant, while the displacement is unique only to within an arbitrary rigid body displacement.

\subsection{Uniqueness in linear thermoelastostatics}

The discussion of uniqueness for linear theories is similar to that for the linearised theory with one important exception. The coefficients $d_{i j k l}$ are replaced by the fully symmetric elasticities $c_{i j k l}$ that satisfy (2.5). Instead of using (2.6) in the operations leading to the energy equation (3.1), we combine (2.25) with (2.7) to obtain for zero source terms the expression

$$
\int_{\Omega}\left(c_{i j k l} e_{i j} e_{k l}+\beta_{i j} u_{i, j} \theta+\Gamma h_{i j k} u_{j, k} \theta_{, i}+\Gamma a_{i} \theta_{, i} \theta+\Gamma k_{i j} \theta_{, i} \theta_{, j}\right) d x=0,
$$

where $e_{i j}$ is defined by (2.23). While other assumptions and notation are unaltered, the positive-definite condition (2.13) is replaced by (2.26). The restriction of the quadratic form in (2.26) to symmetric tensors necessitates the introduction of Korn's inequality given by

$$
\int_{\Omega} u_{i, j} u_{i, j} d x \leq C \int_{\Omega} e_{i j} e_{i j} d x
$$

where $C \geq 1$ is a positive constant dependent upon $\Omega$. It is known (see, for example, $\left[5\right.$, Sect.13]) that when $u_{i}$ vanishes on the boundary $\partial \Omega$ then $C=2$, but otherwise $u_{i}$ must be normalised by the condition

$$
\int_{\Omega}\left(u_{i, j}-u_{j, i}\right) d x=0 .
$$

The uniqueness proof for the Dirichlet problem is easily adapted from that presented before, provided $c_{0}$ replaces $d_{1}$. For the Neumann problem, in order to use Korn's inequality, the solution must be normalised to exclude rigid body displacements (satisfied by (3.8)), and arbitrary additive constants in the temperature, but otherwise is unchanged. 


\section{Spatial stability in linearised thermoelastostat- ics}

\subsection{General introductory comments}

Spatial behaviour of an equilibrium solution is analogous to time evolution of a dynamic solution. Indeed, Kirchhoff's exact analogy between a deformed rod in equilibrium and a spinning top (see [17]) may be generalised, for example, to a nonlinear elastostatic finite or semi-infinite three-dimensional prismatic cylinder in equilibrium subject to zero body forces and homogeneous lateral boundary conditions. In general, the equilibrium equations possess a quasiHamiltonian structure with respect to a preferred spatial variable that serves as a surrogate time variable. The alternative decay or growth of solutions described by the classic Phragmén-Lindelöf principle in elliptic (equilibrium) equations corresponds to Liapunov (asymptotic) stability and instability. In particular, decay relates to Saint-Venant's principle, and measures the persistence of effects due to boundary data and singularities. The reader may consult [10, Sect. 6.2] and [14, Sects. 14.6, 19.1.4] for further comment and references to the literature.

Different techniques, reviewed in [1] and [19], have been developed to investigate the more general problem of continuous data dependence, while particular methods for spatial behaviour are reviewed in $[9,7,8]$. Few if any of these treatments have been applied to thermoelastic problems, especially those considered here. The approach introduced in [4], and extended to classic linear thermoelastostatics in [18], is here selected to treat the semi-infinite prismatic cylinder $\Omega=D \times[0, \infty)$, where the cross-section $D \subset \mathbb{R}^{2}$ is a bounded domain which has boundary $\partial D$ sufficiently smooth to admit application of the divergence theorem. We suppose that $\Omega$ is in equilibrium under zero source terms, and although the lateral surface may be subject to other standard types of boundary conditions, we study the homogeneous lateral boundary data specified by

$$
u_{i}(x)=\theta(x)=0, \quad x \in \partial D \times[0, \infty),
$$

together with the assumption that the displacement $u_{i}\left(x_{\alpha}, 0\right)$ and temperature $\theta\left(x_{\alpha}, 0\right)$ on the base $D(0)$ are pointwise prescribed to be

$$
u_{i}\left(x_{\alpha}, 0\right)=w_{i}\left(x_{\alpha}\right), \quad \theta\left(x_{\alpha}, 0\right)=\chi\left(x_{\alpha}\right), \quad x_{\alpha} \in D(0),
$$

where $w_{i}, \chi$ are given functions that vanish on $\partial D(0)$.

The discussion in Section 4.2 elaborates and amends that presented in [15], and itself is a simplified version of that subsequently developed in Section 5 for the corresponding problem in linear thermoelastostatics. The latter treatment has not been published before.

\subsection{Differential inequality}

We construct an ordinary differential inequality for cross-sectional energy fluxes, rather than for volume measures of energy. In addition to conditions (2.12), 
(2.13), and simple modification of (2.14), we assume that the elasticity and heat conduction tensors are upper bounded on $\Omega$, which permits the introduction of bounded positive constants $\widetilde{d}, \widetilde{k}$ defined by

$$
\begin{aligned}
\widetilde{d}^{2} & =\max _{\Omega} d_{i j k l} d_{i j k l}, \\
\widetilde{k}^{2} & =\max _{\Omega} k_{i j} k_{i j} .
\end{aligned}
$$

An appeal to Cauchy's inequality yields for vectors $\xi \in \mathbb{R}^{3}, \eta \in \mathbb{R}^{3}$

$$
\begin{aligned}
d_{i j k l} \xi_{i} \xi_{k} \eta_{j} \eta_{l} & \leq\left(d_{i j k l} \xi_{i} \xi_{k} d_{p j q l} \xi_{p} \xi_{q}\right)^{1 / 2}\left(\eta_{r} \eta_{r}\right) \\
& \leq\left(d_{i j k l} d_{i j k l}\left(\xi_{p} \xi_{p}\right)^{2}\right)^{1 / 2}\left(\eta_{r} \eta_{r}\right) \\
& \leq \widetilde{d} \xi_{i} \xi_{i} \eta_{j} \eta_{j}, \quad x \in \Omega .
\end{aligned}
$$

Similarly, it may be established that

$$
k_{i j} \xi_{i} \eta_{j} \leq \widetilde{k}\left(\xi_{i} \xi_{i}\right)^{1 / 2}\left(\eta_{j} \eta_{j}\right)^{1 / 2} .
$$

Suppose that the Cartesian coordinate system is such that the $x_{3}$-axis is parallel to the cylinder's axis and the origin is located at a point in the cylinder's base. We consider the linear combination of energy fluxes over a cross-section $D\left(x_{3}\right)$, distance $x_{3}$ from the base, given by

$$
H\left(x_{3}\right)=\int_{D\left(x_{3}\right)} t_{i 3} u_{i} d S+\Lambda \int_{D\left(x_{3}\right)} q_{3} \theta d S
$$

where $\Lambda$ is a positive constant to be determined.

An upper bound for $\left|H\left(x_{3}\right)\right|$ is obtained in part by employing inequalities corresponding to (3.3)-(3.5). Substitution from the constitutive relations (2.3) and (2.4), and subsequent appeal to the Schwarz and Poincaré inequalities, 
together with the upper bounds (4.5) and (4.6), successively yields

$$
\begin{aligned}
& \left|H\left(x_{3}\right)\right| \leq\left|\int_{D\left(x_{3}\right)} t_{i j} n_{j} u_{i} d S\right|+\Lambda\left|\int_{D\left(x_{3}\right)} q_{i} n_{i} \theta d S\right| \\
& =\left|\int_{D\left(x_{3}\right)}\left(d_{i j k l} u_{k, l} u_{i} n_{j}+\beta_{i j} u_{i} n_{j} \theta\right) d S\right| \\
& +\Lambda\left|\int_{D\left(x_{3}\right)}\left(h_{i j k} u_{j, k} n_{i} \theta+a_{i} n_{i} \theta^{2}+k_{i j} \theta_{, j} n_{i} \theta\right) d S\right| \\
& \leq\left[\int_{D\left(x_{3}\right)} d_{i j k l} u_{i, j} u_{k, l} d S \int_{D\left(x_{3}\right)} d_{i j k l} u_{i} n_{j} u_{k} n_{l} d S\right]^{1 / 2} \\
& +\left[\int_{D\left(x_{3}\right)} u_{i} n_{j} u_{i} n_{j} d S \int_{D\left(x_{3}\right)} \beta_{i j} \beta_{i j} \theta^{2} d S\right]^{1 / 2} \\
& +\Lambda\left[\int_{D\left(x_{3}\right)} h_{i j k} u_{j, k} h_{i p q} u_{p, q} d S \int_{D\left(x_{3}\right)} n_{i} n_{i} \theta^{2} d S\right]^{1 / 2} \\
& +\Lambda\left[\int_{D\left(x_{3}\right)}\left(a_{i} a_{i} n_{j} n_{j}\right)^{1 / 2} \theta^{2} d S\right] \\
& +\Lambda\left[\int_{D\left(x_{3}\right)} k_{i j} \theta_{, i} \theta_{, j} d S \int_{D\left(x_{3}\right)} k_{i j} n_{i} n_{j} \theta^{2} d S\right]^{1 / 2} \\
& \leq\left[\widetilde{d}^{2} \int_{D\left(x_{3}\right)} u_{i, j} u_{i, j} d S \int_{D\left(x_{3}\right)} u_{i} u_{i} d S\right]^{1 / 2}+\left[\widetilde{\beta}^{2} \int_{D\left(x_{3}\right)} u_{i} u_{i} d S \int_{D\left(x_{3}\right)} \theta^{2} d S\right]^{1 / 2} \\
& +\Lambda\left[\widetilde{h}^{2} \int_{D\left(x_{3}\right)} u_{i, j} u_{i, j} d S \int_{D\left(x_{3}\right)} \theta^{2} d S\right]^{1 / 2}+\Lambda \widetilde{a} \int_{D\left(x_{3}\right)} \theta^{2} d S \\
& +\Lambda \widetilde{k}\left[\int_{D\left(x_{3}\right)} \theta_{, i} \theta_{, i} d S \int_{D\left(x_{3}\right)} \theta^{2} d S\right]^{1 / 2} \\
& \leq\left(\frac{\widetilde{d}^{2}}{\lambda_{1}}\right)^{1 / 2} \int_{D\left(x_{3}\right)} u_{i, j} u_{i, j} d S \\
& +\left[\frac{\widetilde{\beta}}{\lambda_{1}}+\Lambda\left(\frac{\widetilde{h}^{2}}{\lambda_{1}}\right)^{1 / 2}\right]\left[\int_{D\left(x_{3}\right)} u_{i, j} u_{i, j} d S \int_{D\left(x_{3}\right)} \theta_{, i} \theta_{, i} d S\right]^{1 / 2} \\
& +\Lambda\left[\frac{\widetilde{a}}{\lambda_{1}}+\frac{\widetilde{k}}{\lambda_{1}^{1 / 2}}\right] \int_{D\left(x_{3}\right)} \theta_{, i} \theta_{, i} d S,
\end{aligned}
$$

in which $\lambda_{1}$ denotes the first eigenvalue in the fixed membrane problem for the uniform cross-section $D$, and $\Lambda$ is still to be chosen. 
Application of the arithmetic-geometric mean inequality next gives

$$
\left|H\left(x_{3}\right)\right| \leq c_{3} \int_{D\left(x_{3}\right)} u_{i, j} u_{i, j} d S+\Lambda c_{4} \int_{D\left(x_{3}\right)} \theta_{, i} \theta_{, i} d S
$$

where the constants $c_{3}, c_{4}$ are given by

$$
\begin{aligned}
c_{3} & =\left(\left(\frac{\widetilde{d}^{2}}{\lambda_{1}}\right)^{1 / 2}+\frac{\alpha_{4}}{2} \frac{\widetilde{\beta}}{\lambda_{1}}+\frac{\alpha_{5} \Lambda}{2}\left(\frac{\widetilde{h}^{2}}{\lambda_{1}}\right)^{1 / 2}\right), \\
c_{4} & =\left[\frac{1}{2 \alpha_{4} \Lambda} \frac{\widetilde{\beta}}{\lambda_{1}}+\frac{1}{2 \alpha_{5}}\left(\frac{\widetilde{h}^{2}}{\lambda_{1}}\right)^{1 / 2}+\frac{\widetilde{a}}{\lambda_{1}}+\frac{\widetilde{k}}{\lambda_{1}^{1 / 2}}\right] .
\end{aligned}
$$

Among a variety of possible choices that lead to $c_{3}=c_{4}$, we select the arbitrary positive constants $\alpha_{4}$ and $\alpha_{5}$ to satisfy

$$
\begin{aligned}
\frac{\tilde{d}}{\lambda_{1}^{1 / 2}+\frac{\alpha_{4} \widetilde{\beta}}{2 \lambda_{1}}} & =\frac{\widetilde{\beta}}{2 \alpha_{4} \Lambda \lambda_{1}}, \\
\frac{\alpha_{5} \Lambda \widetilde{h}}{2 \lambda_{1}^{1 / 2}} & =\frac{\widetilde{h}}{2 \alpha_{5} \lambda_{1}^{1 / 2}}+\frac{b}{\lambda_{1}^{1 / 2}},
\end{aligned}
$$

where

$$
b=\left(\frac{\widetilde{a}}{\lambda_{1}^{1 / 2}}+\widetilde{k}\right) .
$$

Appropriate solutions to these equations are

$$
\begin{aligned}
& \alpha_{4}=\frac{1}{\widetilde{\beta}}\left[\left(\widetilde{d}^{2}+\widetilde{\beta}^{2} \Lambda^{-1}\right)^{1 / 2}-\widetilde{d}\right], \\
& \alpha_{5}=\frac{1}{\Lambda \widetilde{h}}\left[\sqrt{\left(b^{2}+\Lambda \widetilde{h}^{2}\right)}+b\right],
\end{aligned}
$$

which lead to the sought upper bound

$$
\left|H\left(x_{3}\right)\right| \leq c_{3}\left(\int_{D\left(x_{3}\right)} u_{i, j} u_{i, j} d S+\Lambda \int_{D\left(x_{3}\right)} \theta_{, i} \theta_{, i} d S\right),
$$

where the positive constant $c_{3}$ is given by

$$
c_{3}=\frac{1}{2 \lambda_{1}^{1 / 2}}\left[\widetilde{d}+\left(\widetilde{d}^{2}+\widetilde{\beta}^{2} \Lambda^{-1} \lambda_{1}^{-1}\right)^{1 / 2}+b+\left(b^{2}+\Lambda \widetilde{h}^{2}\right)^{1 / 2}\right] .
$$

Next, integration by parts and use of (2.6), (2.7) with zero source terms, and the homogeneous lateral boundary conditions (4.1) leads to the expressions

$$
\begin{aligned}
H\left(x_{3}\right)-H\left(y_{3}\right) & =\int_{y_{3}}^{x_{3}} I(\eta) d \eta, \quad 0 \leq y_{3}<x_{3} \leq \infty, \\
H^{\prime}\left(x_{3}\right) & =I\left(x_{3}\right),
\end{aligned}
$$


where a superposed prime indicates differentiation with respect to $x_{3}$, and

$$
I\left(x_{3}\right)=\int_{D\left(x_{3}\right)} B\left(u_{i, j}, \theta_{, i}, \theta\right) d S .
$$

The quadratic form $B$ is given by (3.2) but with $\Gamma$ replaced by $\Lambda$. Subject to (2.12)-(2.17) with $\lambda_{1}$ written for $\lambda_{1}(\Omega)$, we may perform operations similar to those leading to (3.6) to obtain

$$
I\left(x_{3}\right) \geq \int_{D\left(x_{3}\right)}\left(c_{5} u_{i, j} u_{i, j}+\Lambda c_{6} \theta_{, i} \theta_{, i}\right) d S,
$$

where the constants $c_{5}$ and $c_{6}$ are defined by

$$
\begin{aligned}
& c_{5}=\left(d_{1}-\frac{\alpha_{6} \widetilde{\beta}^{2}}{2 \lambda_{1}}-\frac{\alpha_{7} \Lambda \widetilde{h}^{2}}{2}\right), \\
& c_{6}=\left(A-\frac{1}{2 \Lambda \alpha_{6}}-\frac{1}{2 \alpha_{7}}\right),
\end{aligned}
$$

where $\alpha_{6}, \alpha_{7}$ are arbitrary positive constants, and $A>0$ is given by (2.14), but with $\lambda_{1}(\Omega)$ replaced by $\lambda_{1}$.

Set

$$
\frac{\alpha_{6} \widetilde{\beta}^{2}}{\lambda_{1}}=\alpha_{7} \Lambda \widetilde{h}^{2}=\alpha_{8}
$$

and select $\Lambda, \alpha_{8}$ to ensure that the coefficients $c_{5}, c_{6}$ are positive and satisfy $c_{5}=c_{6}$. In view of assumptions (2.12)-(2.14), we choose

$$
\begin{aligned}
\Lambda & =\frac{\widetilde{\beta}}{\lambda_{1}^{1 / 2} \widetilde{h}}, \\
Q & =d_{1}-A,
\end{aligned}
$$

so that $\alpha_{8}$ satisfies the quadratic equation

$$
\alpha_{8}^{2}-Q \alpha_{8}-\widetilde{\beta h} \lambda_{1}^{-1 / 2}=0,
$$

whose positive root is given by

$$
\alpha_{8}=\frac{1}{2}\left[Q+\sqrt{\left(Q^{2}+4 \widetilde{\beta} h \lambda_{1}^{-1 / 2}\right)}\right] .
$$

The condition corresponding to (2.20) implies

$$
\left(A+d_{1}\right)^{2}-\left(d_{1}-A\right)^{2}=4 d_{1} A>4 \widetilde{\beta} \tilde{h} \lambda^{-1 / 2},
$$

and consequently

$$
\begin{aligned}
\sqrt{\left(Q^{2}+4 \widetilde{\beta} \widetilde{h} \lambda^{-1 / 2}\right)} & <A+d_{1} \\
& =\left(A-d_{1}\right)+2 d_{1}
\end{aligned}
$$


which from (4.13) implies $\alpha_{8}<d_{1}$. Subject to these conditions, we have

$$
\begin{aligned}
c_{5} & =d_{1}-\alpha_{8} \\
& =\frac{1}{2}\left[\left(d_{1}+A\right)-\sqrt{\left(Q^{2}+4 \widetilde{\beta h} \lambda_{1}^{-1 / 2}\right)}\right] .
\end{aligned}
$$

We conclude that

$$
H^{\prime}\left(x_{3}\right)=I\left(x_{3}\right) \geq c_{5} \int_{D\left(x_{3}\right)}\left(u_{i, j} u_{i, j}+\Lambda \theta_{, i} \theta_{, i}\right) d S \geq 0, \quad x_{3} \geq 0 .
$$

On combining (4.9) and (4.14), we obtain the required fundamental differential inequality in the form

$$
\left|H\left(x_{3}\right)\right| \leq \alpha^{-1} H^{\prime}\left(x_{3}\right), \quad 0 \leq x_{3} \leq \infty,
$$

where the constant $\alpha=c_{5} / c_{3}$ is positive.

\subsection{Growth and decay estimates}

To extract information from (4.15), we employ the method developed in [4], and first suppose there exists $y_{3} \geq 0$ such that $H\left(y_{3}\right)>0$, which by (4.14) implies that $H\left(x_{3}\right)>0$ for $x_{3} \geq y_{3} \geq 0$. The appropriate component of the fundamental inequality (4.15) becomes

$$
\alpha H\left(x_{3}\right)-H^{\prime}\left(x_{3}\right) \leq 0, \quad y_{3} \leq x_{3} \leq \infty,
$$

which on integration yields

$$
H\left(x_{3}\right) \geq H\left(y_{3}\right) \exp \alpha\left(x_{3}-y_{3}\right), \quad y_{3} \leq x_{3} \leq \infty .
$$

We conclude that $H\left(x_{3}\right) \rightarrow \infty$ as $x_{3} \rightarrow \infty$. We prove that this result leads in turn to the asymptotic unboundedness (as $x_{3} \rightarrow \infty$ ) of the energy function $E\left(y_{3}, x_{3}\right)$ defined to be

$$
E\left(y_{3}, x_{3}\right)=\int_{\Omega\left(y_{3}, x_{3}\right)}\left(u_{i, j} u_{i, j}+\Lambda \theta_{, i} \theta_{, i}\right) d x, \quad 0 \leq y_{3} \leq x_{3} \leq \infty,
$$

where

$$
\Omega\left(y_{3}, x_{3}\right)=\left\{z \in \Omega: y_{3} \leq z_{3} \leq x_{3}\right\},
$$

and $\Lambda$ is specified by (4.12).

It follows from (4.9) that for $0 \leq y_{3} \leq x_{3} \leq \infty$, we have

$$
\begin{aligned}
c_{3} E\left(y_{3}, x_{3}\right) & \geq \int_{y_{3}}^{x_{3}} H(\eta) d \eta \\
& \geq \frac{H\left(y_{3}\right)}{\alpha}\left(\exp \alpha\left(x_{3}-y_{3}\right)-1\right),
\end{aligned}
$$


which proves the assertion on letting $x_{3} \rightarrow \infty$.

On the other hand, let us suppose that the function $E\left(x_{3}, \infty\right)$ is bounded for all $x_{3} \geq 0$, so that $H\left(x_{3}\right) \leq 0,0 \leq x_{3} \leq \infty$. Then, from (4.15) we obtain

$$
H^{\prime}\left(x_{3}\right)+\alpha H\left(x_{3}\right) \geq 0, \quad 0 \leq x_{3} \leq \infty,
$$

which after integration gives

$$
-H\left(x_{3}\right) \leq-H(0) \exp \left(-\alpha x_{3}\right), \quad 0 \leq x_{3} \leq \infty .
$$

We conclude that $H\left(x_{3}\right) \rightarrow 0$ as $x_{3} \rightarrow \infty$. We may alternatively express (4.19) in terms of the energy function $E\left(y_{3}, x_{3}\right)$, defined in (4.17), on first noting from (4.10) that

$$
-H\left(x_{3}\right)=\int_{x_{3}}^{\infty} I(\eta) d \eta,
$$

so that by (4.14) and (4.17) we have

$$
-H\left(x_{3}\right) \geq c_{5} E\left(x_{3}, \infty\right) .
$$

Again, integration by parts applied to (4.7) yields

$$
\begin{aligned}
-H(0) & =\int_{D(0)} t_{i j} u_{i} n_{j} d S+\Lambda \int_{D(0)} q_{i} n_{i} \theta d S \\
& =\int_{\Omega} t_{i j} u_{i, j} d x+\Lambda \int_{\Omega} q_{i} \theta_{, i} d x,
\end{aligned}
$$

where $\Lambda$ is still given by (4.12). Calculations similar to those used to deduce (4.9), show that after substitution from (2.3) and (2.4) we obtain from (4.20) the bound

$$
\begin{aligned}
-H(0)= & \int_{\Omega}\left(d_{i j k l} u_{i, j} u_{k, l}+\beta_{i j} u_{i, j} \theta\right) d x \\
& +\Lambda \int_{\Omega}\left(h_{i j k} u_{j, k} \theta_{, i}+a_{i} \theta \theta_{, i}+k_{i j} \theta_{, i} \theta_{, j}\right) d x \\
\leq & \widetilde{d} \int_{\Omega} u_{i, j} u_{i, j} d x+\widetilde{\beta}\left[\int_{\Omega} u_{i, j} u_{i, j} d x \int_{\Omega} \theta^{2} d x\right]^{1 / 2} \\
& +\Lambda \widetilde{h}\left[\int_{\Omega} u_{i, j} u_{i, j} d x \int_{\Omega} \theta_{, i} \theta_{, i} d x\right]^{1 / 2}+\Lambda \widetilde{a}\left[\int_{\Omega} \theta^{2} d x \int_{\Omega} \theta_{, i} \theta_{, i} d x\right]^{1 / 2} \\
& +\Lambda \widetilde{k} \int_{\Omega} \theta_{, i} \theta_{, i} d x .
\end{aligned}
$$


Poincaré's inequality yields

$$
\begin{aligned}
\int_{\Omega} \theta^{2} d x & =\int_{0}^{\infty} \int_{D(\eta)} \theta^{2} d S d \eta \\
& \leq \int_{0}^{\infty}\left(\lambda_{1}^{-1} \int_{D(\eta)} \theta_{, \alpha} \theta_{, \alpha} d S\right) d \eta \\
& \leq \int_{0}^{\infty}\left(\lambda_{1}^{-1} \int_{D(\eta)} \theta_{, i} \theta_{, i} d S\right) d \eta \\
& =\lambda_{1}^{-1} \int_{\Omega} \theta_{, i} \theta_{, i} d x
\end{aligned}
$$

which on application of the arithmetic-geometric mean inequality leads to

$$
-H(0)=c_{7} \int_{\Omega} u_{i, j} u_{i, j} d x+\Lambda c_{8} \int_{\Omega} \theta_{, i} \theta_{, i} d x,
$$

where $\Lambda$ is specified by (4.12), and for arbitrary positive constant $\alpha_{9}$,

$$
\begin{aligned}
& c_{7}=\left[\widetilde{d}+\frac{L \alpha_{9}}{2}\right], \\
& c_{8}=\left[\frac{1}{2 \alpha_{9}}\left(\frac{(1+\Lambda)}{\Lambda}\right)+b\right] .
\end{aligned}
$$

The constant $b$ is given by (4.8) while the constant $L$ is given by

$$
\begin{aligned}
L & =\frac{\widetilde{\beta}^{2}}{\lambda_{1}}+\Lambda \widetilde{h}^{2} \\
& =(1+\Lambda) \frac{\widetilde{\beta} \widetilde{h}}{\lambda_{1}^{1 / 2}} .
\end{aligned}
$$

We choose $\alpha_{9}$ such that $c_{7}=c_{8}$, and consequently have

$$
\alpha_{9}^{2} L+2 \alpha_{9}(\widetilde{d}-b)-\left(1+\Lambda^{-1}\right)=0,
$$

whose positive root is

$$
\alpha_{9}=\frac{(b-\widetilde{d})+\sqrt{(b-\widetilde{d})^{2}+\widetilde{h}^{2}(1+\Lambda)^{2}}}{L} .
$$

We conclude that

$$
c_{7}=\frac{1}{2}\left[\widetilde{d}+b+\sqrt{(b-\widetilde{d})^{2}+\widetilde{h}^{2}(1+\Lambda)^{2}}\right]
$$

and finally that

$$
-H(0) \leq c_{7} E(0, \infty)
$$


Consequently, we may rewrite (4.19) as

$$
c_{5} E\left(x_{3}, \infty\right) \leq-H\left(x_{3}\right) \leq c_{7} E(0, \infty) \exp \left(-\alpha x_{3}\right) .
$$

We have proved:

Theorem 4.1 Let us assume that conditions (I) and (II) are satisfied and that inequalities (2.14), (2.17), (4.5) and (4.6) hold. Then on the prismatic semiinfinite cylinder, the solution to the linear system (2.6) and (2.7) subject to zero source terms and homogeneous lateral boundary conditions (4.1) satisfies either the growth estimate (4.16) or the decay estimates (4.19) and (4.21).

Uniqueness is an immediate corollary of this Theorem.

Corollary 4.1 (Uniqueness) In the class of bounded energies $E(0, \infty)$ there is at most one solution to the boundary value problem (2.6), (2.7) on the semiinfinite prismatic cylinder subject to conditions stipulated in Theorem 4.1.

Proof: First, note that by hypothesis, $H\left(x_{3}\right) \leq 0$ for $x_{3} \geq 0$. It must be proved that at most only the trivial solution exists for homogeneous data. Thus, we assume that $H(0)=0$ and from (4.19) deduce that $H\left(x_{3}\right)=0$ for $0 \leq x_{3} \leq \infty$, which by (4.21) implies $E\left(x_{3}, \infty\right)=0, x_{3} \geq 0$. Consequently, $u_{i, j}=\theta_{, i}=0$ for $x \in D \times[0, \infty)$ and so by continuity and the boundary conditions, we conclude that $u_{i}=\theta=0$ for $x \in D \times[0, \infty)$. The proof is complete.

\subsection{The amplitude term}

A full description of the decay estimate requires an upper bound for the amplitudes, $-H(0)$ or $E(0)$, in terms of base data.

For this purpose, we introduce smooth functions $v_{i}(x), \phi(x)$ that satisfy the boundary conditions

$$
\begin{aligned}
& v_{i}(x)=\phi(x)=0, \quad x \in \partial D \times[0, \infty), \\
& v_{i}(x)=w_{i}\left(x_{\alpha}\right), \quad \phi(x)=\chi\left(x_{\alpha}\right), \quad x \in D(0),
\end{aligned}
$$

where the Dirichlet base data functions are specified in (4.2). We also suppose the asymptotic behaviour

$$
v_{i}(x) \rightarrow 0, \quad \phi(x) \rightarrow 0, \quad x_{3} \rightarrow \infty
$$

and that all source terms vanish.

On multiplying (2.6) by $v_{i}$, adding to (2.7) multiplied by $\Lambda \phi$ for positive constant $\Lambda$ defined by (4.12), integrating by parts over $\Omega$, and recalling (4.11), we obtain after some rearrangement

$$
\begin{aligned}
c_{5} E(0, \infty) & \leq \int_{\Omega} B d x \\
& =\int_{\Omega}\left(d_{i j k l} v_{i, j} u_{k, l}+\beta_{i j} \theta v_{i, j}+\Lambda h_{i j k} u_{j, k} \phi_{, i}+\Lambda a_{i} \theta \phi_{, i}+\Lambda k_{i j} \theta_{, j} \phi_{, i}\right) d x .
\end{aligned}
$$


Standard use of the Schwarz, Poincaré, and arithmetic-geometric mean inequalities next yields

$$
c_{5} E(0, \infty) \leq c_{9} \int_{\Omega} v_{i, j} v_{i, j} d x+\Lambda c_{10} \int_{\Omega} \phi_{, i} \phi_{, i} d x
$$

where

$$
\begin{aligned}
c_{9} & =\left(\frac{\widetilde{d}^{2} \alpha_{10}}{2}+\frac{\widetilde{\beta}^{2} \alpha_{11}}{2 \lambda_{1}}\right), \\
c_{10} & =\left(\frac{\Lambda \widetilde{h}^{2} \alpha_{12}}{2}+\frac{\widetilde{a}^{2} \alpha_{13}}{2 \lambda_{1}}+\frac{\widetilde{k}^{2} \alpha_{14}}{2}\right),
\end{aligned}
$$

in which $\widetilde{d}$ and $\widetilde{k}$ are defined in (4.3) and (4.4) respectively, and the arbitrary positive constants $\alpha_{i}, i=10, \ldots 14$ are chosen to be

$$
\begin{aligned}
\alpha_{10} & =\alpha_{12}=2 c_{5}^{-1}, \\
\Lambda \alpha_{11} & =\alpha_{13}=\alpha_{14}=3 c_{5}^{-1} .
\end{aligned}
$$

Hence, we have

$$
\begin{aligned}
c_{9} & =\frac{1}{2 \lambda_{1} \Lambda c_{5}}\left(2 \lambda_{1} \Lambda \widetilde{d}^{2}+3 \widetilde{\beta}^{2}\right), \\
c_{10} & =\frac{1}{2 \lambda_{1} c_{5}}\left(2 \lambda_{1} \Lambda \widetilde{h}^{2}+3 \widetilde{a}^{2}+3 \lambda_{1} \widetilde{k}^{2}\right)
\end{aligned}
$$

We now select the functions $v_{i}, \phi$ to be

$$
\begin{aligned}
v_{i}(x) & =u_{i}\left(x_{\alpha}, 0\right) \exp \left(-s x_{3}\right)=w_{i}\left(x_{\alpha}\right) \exp \left(-s x_{3}\right), \\
\phi(x) & =\theta\left(x_{\alpha}, 0\right) \exp \left(-s x_{3}\right)=\chi\left(x_{\alpha}\right) \exp \left(-s x_{3}\right),
\end{aligned}
$$

where $s$ is a positive constant to be determined, and the functions $w_{i}, \chi$ are prescribed. Substitution in (4.25) and optimisation with respect to $s$ leads to the required estimate

$$
c_{5} E(0, \infty) \leq\left(D_{1} D_{2}\right)^{1 / 2}
$$

where the data terms $D_{1}, D_{2}$ are given by

$$
\begin{aligned}
D_{1} & =c_{9} \int_{D} w_{i, \beta} w_{i, \beta} d S+\Lambda c_{10} \int_{D} \chi_{, \beta} \chi_{, \beta} d S \\
D_{2} & =c_{9} \int_{D} w_{i} w_{i} d S+\Lambda c_{10} \int_{D} \chi^{2} d S .
\end{aligned}
$$

\subsection{Additional decay behaviour}

We may deduce from inequality (4.21) that the mean-square over $D\left(x_{3}\right)$ of the displacement and temperature, and of the displacement and temperature 
gradient, exhibit similar exponential decay. Note also that the derivation of the basic inequality (4.15) is valid irrespective of the cylinder's length, and in particular holds for an infinite cylinder. We infer that on the infinite cylinder only the trivial solution can exist in the class of bounded stored energies.

The previous analysis includes the special case of a uniform primary temperature when $\widetilde{h}=\widetilde{a}=0$. In consequence, only inequalities (2.12), (2.13), (4.5), and (4.6) are needed to establish conclusions similar to those just derived.

\section{$5 \quad$ Spatial stability for linear thermoelastostatics}

\section{$5.1 \quad$ Introduction}

We now investigate spatial stability for the linear theory of thermoelastostatics represented by the system (2.25) and (2.11) subject to appropriate boundary conditions. For simplicity, we continue to consider the semi-infinite prismatic cylinder $\Omega=D \times[0, \infty)$, whose lateral boundary $\partial D \times[0, \infty)$ is sufficiently smooth to admit application of the divergence theorem. A Cartesian system of rectangular coordinates is again chosen with origin in the base of the cylinder and positive $x_{3}$-axis directed along that of the cylinder. In addition, $D\left(x_{3}\right)$ denotes the cross-section at distance $x_{3}$ from the cylinder's base, and we define partial volumes $\Omega\left(y_{3}, x_{3}\right)$ of $\Omega$ by (4.18). In this Section, however, we suppose that $\Omega$ is occcupied by a linear thermoelastic material in equilibrium under zero body force and heat supply, and loaded only on the base $D(0)$ with the remaining lateral surface subject to the homogeneous data

$$
\begin{array}{rlrl}
t_{i \alpha} n_{\alpha} u_{i} & =0, & & x \in \partial D \times[0, \infty), \\
k_{\alpha \beta} \theta_{, \alpha} n_{\beta} \theta & =0, & x \in \partial D \times[0, \infty),
\end{array}
$$

where $n_{i}$ are the Cartesian components of the unit outward normal on $\partial \Omega$.

The linear elasticities $c_{i j k l}$ satisfy the major and minor symmetries (2.5) and are positive-definite in the sense of inequality (2.26). Likewise, we suppose that the heat conduction tensor $k_{i j}$ satisfies the positive-definite condition (2.12).

Proofs are completed in detail only for homogeneous Dirichlet data on the lateral surface, while those for Neumann data are only sketched. It should be emphasised that asymptotic behaviour is not prescribed for large axial distance, but, as in Section 4, emerges as a consequence of the arguments. As before, we establish and integrate a differential inequality for the cross-sectional energy flux $H\left(x_{3}\right)$, defined in (4.7), but with the constant $\Lambda$ not necessarily given by (4.12). Significant modification of the method described in Section 4.2 is required due to the positive condition (2.26) being restrictied to symmetric tensors.

The special example of a nonhomogeneous isotropic linear thermoelastic rectangular strip considered in [2] uses the Airy stress function and second order differential inequalities to establish decay rates by a different method to that developed here. 


\subsection{Differential inequality}

Consider the previously introduced cross-sectional flux (4.7), repeated here for convenience,

$$
H\left(x_{3}\right)=\int_{D\left(x_{3}\right)} t_{i 3} u_{i} d S+\Lambda \int_{D\left(x_{3}\right)} q_{3} \theta d S,
$$

in which the positive constant $\Lambda$ is not specified by (4.12). Integration by parts over $\Omega\left(y_{3}, x_{3}\right)$, appeal to the equilibrium equations (2.25) and (2.11), subject to vanishing body force and heat supply, and the lateral boundary conditions (5.1) and (5.2), successively yields

$$
\begin{aligned}
H\left(x_{3}\right)-H\left(y_{3}\right)= & \int_{\Omega\left(y_{3}, x_{3}\right)}\left(t_{i 3} u_{i}\right)_{, 3} d x+\Lambda \int_{\Omega\left(y_{3}, x_{3}\right)}\left(q_{3} \theta\right)_{, 3} d x \\
= & \int_{\Omega\left(y_{3}, x_{3}\right)}\left(-t_{i \alpha, \alpha} u_{i}+t_{i 3} u_{i, 3}\right) d x \\
& +\Lambda \int_{\Omega\left(y_{3}, x_{3}\right)}\left(-q_{\alpha, \alpha} \theta+q_{3} \theta_{, 3}\right) d x \\
= & \int_{\Omega\left(y_{3}, x_{3}\right)} t_{i j} u_{i, j} d x+\Lambda \int_{\Omega\left(y_{3}, x_{3}\right)} q_{i} \theta_{, i} d x+H\left(y_{3}\right) \\
= & \int_{\Omega\left(y_{3}, x_{3}\right)}\left(c_{i j k l} e_{k l} u_{i, j}+\beta_{i j} u_{i, j} \theta\right) d x+\Lambda \int_{\Omega\left(y_{3}, x_{3}\right)} k_{i j} \theta_{, i} \theta_{, j} d x \\
= & \int_{\Omega\left(y_{3}, x_{3}\right)}\left(c_{i j k l} e_{i j} e_{k l}+\beta_{i j} e_{i j} \theta\right) d x \\
& +\Lambda \int_{\Omega\left(y_{3}, x_{3}\right)} k_{i j} \theta_{, i} \theta_{, j} d x
\end{aligned}
$$

where the symmetries (2.5) and (2.24) are employed. Differentiation of (5.4) with respect to $x_{3}$ gives

$$
H^{\prime}\left(x_{3}\right)=\int_{D\left(x_{3}\right)}\left(c_{i j k l} e_{i j} e_{k l}+\beta_{i j} e_{i j} \theta\right) d S+\Lambda \int_{D\left(x_{3}\right)} k_{i j} \theta_{, i} \theta_{, j} d S,
$$

in which we recall that a superposed prime indicates differentiation with respect to the argument. The Poincaré and the arithmetic-geometric mean inequalities lead to the lower bound

$$
H^{\prime}\left(x_{3}\right) \geq\left(1-\frac{\gamma_{1}}{2}\right) \int_{D\left(x_{3}\right)} c_{i j k l} e_{i j} e_{k l} d S+\left(\Lambda-\frac{\widetilde{\beta}^{2}}{2 \gamma_{1} c_{0} k_{1} \lambda_{1}}\right) \int_{D\left(x_{3}\right)} k_{i j} \theta_{, i} \theta_{, j} d S
$$

where $\gamma_{1}$ is an arbitrary positive constant, $k_{1}, \widetilde{\beta}$, and $c_{0}$ are defined in (2.12), (2.18), and (2.26), respectively, and $\lambda_{1}$ denotes the first eigenvalue for the fixed membrane problem for the uniform cross-section $D$. On setting

$$
\gamma_{1}^{2}=\frac{\widetilde{\beta}^{2}}{\Lambda c_{0} k_{1} \lambda_{1}}
$$


and choosing $\Lambda$ such that

$$
0<\gamma_{1}<2
$$

we conclude that the lower bound (5.6) becomes

$$
H^{\prime}\left(x_{3}\right) \geq\left(1-\frac{\gamma_{1}}{2}\right)\left[\int_{D\left(x_{3}\right)} c_{i j k l} e_{i j} e_{k l} d S+\Lambda \int_{D\left(x_{3}\right)} k_{i j} \theta_{, i} \theta_{, j} d S\right]
$$

In consequence of (2.12) and (2.26), it follows that

$$
H^{\prime}\left(x_{3}\right) \geq 0, \quad 0 \leq x_{3} \leq \infty,
$$

and by integration that

$$
H\left(x_{3}\right) \geq\left(1-\frac{\gamma_{1}}{2}\right) E\left(y_{3}, x_{3}\right)+H\left(y_{3}\right),
$$

where the energy $E\left(y_{3}, x_{3}\right)$ is defined to be

$$
E\left(y_{3}, x_{3}\right)=\int_{\Omega\left(y_{3}, x_{3}\right)}\left(c_{i j k l} e_{i j} e_{k l}+\Lambda k_{i j} \theta_{, i} \theta_{, j}\right) d x \geq 0, \quad 0 \leq y_{3} \leq x_{3} \leq \infty .
$$

Note that $H\left(x_{3}\right)$ is not necessarily either positive or negative. Moreover, subject to conditions (5.7) and (5.8), the relations (5.4) and (5.9) generate the upper bound

$$
\begin{aligned}
0 & \leq\left(1-\frac{\gamma_{1}}{2}\right) E\left(y_{3}, x_{3}\right), \quad 0 \leq y_{3} \leq x_{3} \leq \infty \\
& \leq H\left(x_{3}\right)-H\left(y_{3}\right) \\
& =\int_{\Omega\left(y_{3}, x_{3}\right)}\left(c_{i j k l} e_{i j} e_{k l}+\beta_{i j} e_{i j} \theta+\Lambda k_{i j} \theta_{, i} \theta_{, j}\right) d x
\end{aligned}
$$

which is of later use.

The next task is to obtain an upper bound for the absolute value of $H\left(x_{3}\right)$ in terms of $H^{\prime}\left(x_{3}\right)$. Since $n=(0,0,1)$ on $D\left(x_{3}\right)$, we have for all $x_{3} \geq 0$,

$$
\begin{aligned}
\left|H\left(x_{3}\right)\right| \leq & \left|\int_{D\left(x_{3}\right)} t_{i 3} u_{i} d S\right|+\Lambda\left|\int_{D\left(x_{3}\right)} q_{3} \theta d S\right| \\
= & \left|\int_{D\left(x_{3}\right)} t_{i j} n_{j} u_{i} d S\right|+\Lambda\left|\int_{D\left(x_{3}\right)} q_{i} n_{i} \theta d S\right| \\
= & \left|\int_{D\left(x_{3}\right)}\left(c_{i j k l} e_{k l} n_{j} u_{i}+\beta_{i j} n_{j} u_{i} \theta\right) d S\right| \\
& +\Lambda\left|\int_{D\left(x_{3}\right)} k_{i j} \theta,{ }_{, j} n_{i} \theta d S\right|,
\end{aligned}
$$


which, on using Schwarz's inequality, enables us to conclude that

$$
\begin{aligned}
\left|H\left(x_{3}\right)\right| \leq & {\left[\int_{D\left(x_{3}\right)} c_{i j k l} e_{i j} e_{k l} d S \int_{D\left(x_{3}\right)} c_{i j k l} u_{i} u_{k} n_{j} n_{l} d S\right]^{1 / 2} } \\
+ & \widetilde{\beta}\left[\int_{D\left(x_{3}\right)} u_{i} u_{i} d S \int_{D\left(x_{3}\right)} \theta^{2} d S\right]^{1 / 2} \\
+ & {\left[\int_{D\left(x_{3}\right)} k_{i j} \theta_{, i} \theta, d S \int_{D\left(x_{3}\right)} k_{i j} n_{i} n_{j} \theta^{2} d S\right]^{1 / 2} }
\end{aligned}
$$

Furthermore, application of the arithmetic-geometric mean inequality and the Cauchy-Schwarz inequality leads to the bounds

$$
\begin{aligned}
\int_{D\left(x_{3}\right)} c_{i j k l} u_{i} u_{k} n_{j} n_{l} d S & =\int_{D\left(x_{3}\right)} c_{i 3 k 3} u_{i} u_{k} d S \\
& \leq\left[\int_{D\left(x_{3}\right)} c_{i 3 k 3} u_{i} c_{p 3 k 3} u_{p} d S \int_{D\left(x_{3}\right)} u_{k} u_{k} d S\right]^{1 / 2} \\
& \leq\left[\int_{D\left(x_{3}\right)}\left(c_{i 3 k 3} c_{i 3 k 3}\right) u_{i} u_{i} d S \int_{D\left(x_{3}\right)} u_{k} u_{k} d S\right]^{1 / 2} \\
& \leq \widetilde{c} \int_{D\left(x_{3}\right)} u_{i} u_{i} d S
\end{aligned}
$$

where

$$
\widetilde{c}^{2}=\max _{\Omega} c_{i 3 k 3} c_{i 3 k 3} .
$$

Similarly, we have

$$
\begin{aligned}
\int_{D\left(x_{3}\right)} k_{i j} n_{i} n_{j} \theta^{2} d S & =\int_{D\left(x_{3}\right)} k_{33} \theta^{2} d S \\
& \leq k_{3} \int_{D\left(x_{3}\right)} \theta^{2} d S
\end{aligned}
$$

where

$$
k_{3}=\max _{\Omega} k_{33} .
$$

Insertion of the bounds (5.15) and (5.17) into (5.14) succeeded by further 
application of the arithmetic-geometric mean inequality yields

$$
\begin{aligned}
\mid H\left(\left(x_{3}\right) \mid \leq\right. & {\left[\int_{D\left(x_{3}\right)} c_{i j k l} e_{i j} e_{k l} d S \int_{D\left(x_{3}\right)} u_{i} u_{i} d S\right]^{1 / 2} } \\
& +\widetilde{\beta}\left[\int_{D\left(x_{3}\right)} u_{i} u_{i} d S \int_{D\left(x_{3}\right)} \theta^{2} d S\right]^{1 / 2} \\
& +\Lambda\left[k_{3} \int_{D\left(x_{3}\right)} k_{i j} \theta_{, i} \theta_{, j} d S \int_{D\left(x_{3}\right)} \theta^{2} d S\right]^{1 / 2} \\
\leq & \left(\frac{\widetilde{c}^{2} \gamma_{2}}{2}\right) \int_{D\left(x_{3}\right)} c_{i j k l} e_{i j} e_{k l} d S \\
& +\frac{1}{2}\left(\frac{1}{\gamma_{2}}+\frac{1}{\gamma_{3}}\right) \int_{D\left(x_{3}\right)} u_{i} u_{i} d S \\
& +\frac{\widetilde{\beta}^{2} \gamma_{3}}{2} \int_{D\left(x_{3}\right)} \theta^{2} d S+\frac{\Lambda k_{3}^{1 / 2}}{\left(k_{1} \lambda_{1}\right)^{1 / 2}} \int_{D\left(x_{3}\right)} k_{i j} \theta_{, i} \theta_{, j} d S \\
\leq \quad & \left.\frac{\widetilde{c}^{2} \gamma_{2}}{2} \int_{D\left(x_{3}\right)} c_{i j k l} e_{i j} e_{k l} d S+\left(\frac{\widetilde{\beta}^{2} \gamma_{3}}{2 k_{1} \lambda_{1}}+\frac{\Lambda k_{3}^{1 / 2}}{\left(k_{1} \lambda_{1}\right)^{1 / 2}}\right) \int_{D\left(x_{3}\right)} k_{i j} \theta_{, i} \theta_{, j} d S\right\} \\
& +\frac{1}{2}\left(\frac{1}{\gamma_{2}}+\frac{1}{\gamma_{3}}\right) \int_{D\left(x_{3}\right)} u_{i} u_{i} d S,
\end{aligned}
$$

where Poincaré's inequality is used, the constants $\widetilde{\beta}, k_{1}, \lambda_{1}$ have previously been introduced, and $\gamma_{2}, \gamma_{3}$ are arbitrary positive constants chosen to satisfy

$$
\Lambda\left(\frac{\widetilde{c}^{2} \gamma_{2}}{2}\right)=\left(\frac{\widetilde{\beta}^{2} \gamma_{3}}{2 k_{1} \lambda_{1}}+\frac{\Lambda k_{3}^{1 / 2}}{\left(k_{1} \lambda_{1}\right)^{1 / 2}}\right)
$$

Consequently, by virtue additionally of (5.9), (5.18) reduces to

$$
\left|H\left(x_{3}\right)\right| \leq \frac{\widetilde{c}^{2} \gamma_{2}}{\left(2-\gamma_{1}\right)} H^{\prime}\left(x_{3}\right)+\frac{1}{2}\left(\frac{1}{\gamma_{2}}+\frac{1}{\gamma_{3}}\right) \int_{D\left(x_{3}\right)} u_{i} u_{i} d S
$$

In order to bound the second term on the right of this inequality, we appeal to a generalised Korn's inequality stated in the following lemma, whose proof is variously established, for example, in [20], [12], or [3]. The proof, however, given in [12] holds only for sufficiently large $x_{3}$.

Lemma 5.1 Let $\Omega$ be the semi-infinite cylinder defined in this Section, and let $v_{i}(x)$ be a smooth vector function defined on $\Omega$ that satisfies the lateral boundary conditions

$$
v_{i}(x)=0, \quad x \in \partial D \times[0, \infty) .
$$

Then there exists a positive bounded computable positive constant $N$ dependent on the geometry of the (prismatic) cross-section $D$, such that for $0 \leq y_{3}<x_{3} \leq$ 
$\infty$, the following inequality holds

$$
\int_{D\left(y_{3}\right)} v_{i} v_{i} d S+\int_{D\left(x_{3}\right)} v_{i} v_{i} d S \leq N \int_{\Omega\left(y_{3}, x_{3}\right)}\left(v_{i, j}+v_{j, i}\right)\left(v_{i, j}+v_{j, i}\right) d x .
$$

Substitution of (5.21) in (5.20) subsequently leads to the successive inequalities

$$
\begin{aligned}
\left|H\left(x_{3}\right)\right| \leq & \frac{\widetilde{c}^{2} \gamma_{2}}{\left(2-\gamma_{1}\right)} H^{\prime}\left(x_{3}\right)+\frac{1}{2}\left(\frac{1}{\gamma_{2}}+\frac{1}{\gamma_{3}}\right)\left[4 N \int_{\Omega\left(0, x_{3}\right)} e_{i j} e_{i j} d x\right] \\
\leq & \frac{\widetilde{c}^{2} \gamma_{2}}{\left(2-\gamma_{1}\right)} H^{\prime}\left(x_{3}\right)+\frac{1}{2}\left(\frac{1}{\gamma_{2}}+\frac{1}{\gamma_{3}}\right)\left[\frac{4 N}{c_{0}} \int_{\Omega\left(0, x_{3}\right)} c_{i j k l} e_{i j} e_{k l} d x\right] \\
\leq & \frac{\widetilde{c}^{2} \gamma_{2}}{\left(2-\gamma_{1}\right)} H^{\prime}\left(x_{3}\right)+\frac{1}{2}\left(\frac{1}{\gamma_{2}}+\frac{1}{\gamma_{3}}\right) \frac{4 N}{c_{0}} E\left(0, x_{3}\right) \\
\leq & \frac{\widetilde{c}^{2} \gamma_{2}}{\left(2-\gamma_{1}\right)} H^{\prime}\left(x_{3}\right) \\
& +\left(\frac{1}{\gamma_{2}}+\frac{1}{\gamma_{3}}\right) \frac{4 N}{c_{0}\left(2-\gamma_{1}\right)}\left(H\left(x_{3}\right)-H(0)\right),
\end{aligned}
$$

where (2.12), (2.26), and (5.11) are used. The basic differential inequality (5.22) is now employed to establish alternative evolutionary growth and decay properties descriptive of spatial stability. The mutually exclusive initial data for which $H(0)>0$ or $H(0) \leq 0$ require separate discussion.

\subsection{Growth and decay estimates}

We consider first the case for which $H(0)>0$ and prove the following the theorem:

Theorem 5.1 Suppose initial data is such that $H(0)>0$. Then

$$
H\left(x_{3}\right) \geq H(0) \exp \left(\gamma x_{3}\right), \quad 0 \leq x_{3} \leq \infty,
$$

where

$$
\gamma=\frac{\left(2-\gamma_{1}\right)}{2 \widetilde{c}^{2}\left(e \gamma_{3}+f\right)}
$$

and $\gamma_{1}$ and $\widetilde{c}$ are defined in (5.7) and (5.16), while $\gamma_{3}, e, f$ are given by expressions (5.28) and (5.27) below.

Proof: From (5.10), the derivative of $H\left(x_{3}\right)$ with respect to $x_{3}$ is nonnegative, and consequently assumption $H(0)>0$ implies that $H\left(x_{3}\right) \geq H(0)>$ 0 for $x_{3} \geq 0$, so that inequality (5.22) may be written as

$$
H\left(x_{3}\right) \leq \frac{\widetilde{c}^{2} \gamma_{2}}{\left(2-\gamma_{1}\right)} H^{\prime}\left(x_{3}\right)+\left(\frac{1}{\gamma_{2}}+\frac{1}{\gamma_{3}}\right) \frac{4 N}{c_{0}\left(2-\gamma_{1}\right)} H\left(x_{3}\right)
$$


for $0 \leq x_{3} \leq \infty$.

Recall that the arbitrary positive constants $\gamma_{2}, \gamma_{3}$ have been chosen to satisfy condition (5.19), which we rewrite in the form

$$
\gamma_{2}=e \gamma_{3}+f,
$$

where

$$
e=\frac{\widetilde{\beta}^{2}}{\Lambda \widetilde{c}^{2} k_{1} \lambda_{1}}, \quad f=\frac{2 k_{3}^{1 / 2}}{\widetilde{c}^{2}\left(k_{1} \lambda_{1}\right)^{1 / 2}} .
$$

Observe also that the positive constant $\gamma_{1}$ and the constant $\Lambda$ are determined by conditions (5.7) and (5.8). We complete the choice of $\gamma_{2}$ and $\gamma_{3}$ by imposing the condition

$$
2 g\left(\gamma_{2}+\gamma_{3}\right)=\gamma_{2} \gamma_{3},
$$

where

$$
g=\frac{4 N}{c_{0}\left(2-\gamma_{1}\right)} .
$$

Accordingly, $\gamma_{3}$ is given by

$$
\gamma_{3}=\frac{[2 g(1+e)-f]+\sqrt{\left([2 g(1+e)-f]^{2}+8 e f g\right)}}{2 e},
$$

and $\gamma_{2}$ follows from (5.26). We recover (5.23) after substitution in (5.25) followed by integration.

An appeal to identity (5.5) establishes the following corollary to Theorem 5.1, which provides an alternative interpretation of the growth condition.

Corollary 5.1 The total energy (5.12) is unbounded as $x_{3} \rightarrow \infty$ when $H(0)>$ 0 .

Proof: The arithmetic-geometric mean inequality applied to the second term on the right of (5.5) leads to the inequality, compatible with the bound (5.11),

$$
H\left(x_{3}\right) \leq\left[1+\frac{1}{2}\left(\frac{\widetilde{\beta}^{2}}{c_{0} \Lambda \lambda_{1} k_{1}}\right)^{1 / 2}\right] E\left(y_{3}, x_{3}\right)+H\left(y_{3}\right), \quad 0 \leq y_{3}<x_{3} \leq \infty,
$$

which implies the conclusion since we may let $y_{3} \rightarrow 0$ and $x_{3} \rightarrow \infty$.

We next consider the class of displacements and temperatures for which the total energy $E(0, \infty)$ is bounded and immediately have:

Proposition 5.1 Within the class of non-trivial displacements and temperatures, a bounded total energy implies $H\left(x_{3}\right)<0$ for $x_{3} \geq 0$.

Proof: Let us first consider the case $H(0)=0$, and suppose that $H\left(x_{3}\right) \equiv 0$ for all $x_{3} \geq 0$. Then it follows from inequality (5.11) that $e_{i j}=\theta_{, i}=0$ for $x_{3} \geq 0$. Consequently, by virtue of the lateral boundary conditions, the 
corresponding displacement and temperature are identically zero and therefore excluded by assumption. Next suppose that for $H(0)=0$ we have $H(z)>0$ for some $x_{3}=z>0$. Then by Theorem 5.1, the energy is unbounded for $x_{3} \geq z$, contrary to hypothesis. Consequently, we conclude that $H\left(x_{3}\right)<0$ for $x_{3} \geq 0$ whenever the total energy is bounded.

The conditions for Proposition 5.1 are now employed to derive a decay estimate.

Theorem 5.2 In the class of displacements and temperatures that possess bounded total energy, the energy flux function $H\left(x_{3}\right)$ satisfies the differential inequality

$$
0 \leq H^{\prime}\left(x_{3}\right)+\gamma H\left(x_{3}\right), \quad x_{3} \geq 0,
$$

where $\gamma$ is specified by (5.24). Upon integration, we therefore have

$$
-H\left(x_{3}\right) \leq-H(0) \exp \left(-\gamma x_{3}\right), \quad x_{3} \geq 0 .
$$

Proof: We repeat the derivation of inequality (5.22) apart from one, but important, difference.

A total bounded energy implies that $H\left(x_{3}\right)<0, x_{3} \geq 0$, and upon returning to inequality (5.20), we have

$$
-H\left(x_{3}\right) \leq \frac{\widetilde{c}^{2} \gamma_{2}}{\left(2-\gamma_{1}\right)} H^{\prime}\left(x_{3}\right)+\frac{1}{2}\left(\frac{1}{\gamma_{2}}+\frac{1}{\gamma_{3}}\right) \int_{D\left(x_{3}\right)} u_{i} u_{i} d S .
$$

where the constants $\gamma_{1}, \Lambda$ are assumed to satisfy (5.7) and (5.8), and $\gamma_{2}, \gamma_{3}$ are given by (5.28) and (5.26). Now successively apply the bounds (5.21) and (5.11) not to the region $\Omega\left(y_{3}, x_{3}\right)$ but to the region $\Omega\left(x_{3}, z_{3}\right)$ where $0 \leq x_{3}<z_{3}$. We obtain:

$$
\begin{aligned}
\int_{D\left(x_{3}\right)} u_{i} u_{i} d S & \leq \frac{4 N}{c_{0}} \int_{\Omega\left(x_{3}, z_{3}\right)} c_{i j k l} e_{i j} e_{k l} d x \\
& \leq \frac{4 N}{c_{0}} E\left(x_{3}, z_{3}\right) \\
& \leq \frac{8 N}{c_{0}\left(2-\gamma_{1}\right)}\left[H\left(z_{3}\right)-H\left(x_{3}\right)\right] \\
& \leq \frac{8 N}{c_{0}\left(2-\gamma_{1}\right)}\left(-H\left(x_{3}\right)\right)
\end{aligned}
$$

as $H\left(z_{3}\right)<0$. On insertion of (5.33) into (5.32) and after letting $\gamma_{3}$ and $\gamma_{2}$ be determined respectively from (5.28) and (5.26), we conclude that (5.30) holds. Integration leads to (5.31)

The conclusion of Theorem 5.2 may be expressed in terms of the energy $E\left(x_{3}, \infty\right)$. We have:

Proposition 5.2 The energy $E\left(x_{3}, \infty\right)$, defined by (5.12), possesses the exponentially decaying upper bound

$$
E\left(x_{3}, \infty\right) \leq M E(0, \infty) \exp \left(-\gamma x_{3}\right), \quad x_{3} \geq 0,
$$


where the positive constant $M$ is given by

$$
M=\left(1-\frac{\gamma_{1}}{2}\right)^{-1}\left[1+\frac{1}{2}\left(\frac{\widetilde{\beta}^{2}}{c_{0} \Lambda \lambda_{1} k_{1}}\right)^{1 / 2}\right] .
$$

Proof: Let $x_{3} \rightarrow \infty$ in the upper bound (5.31) to conclude that

$$
H\left(x_{3}\right) \rightarrow 0 \quad \text { as } x_{3} \rightarrow \infty,
$$

which upon insertion into inequality (5.11) leads to

$$
-H\left(x_{3}\right) \geq\left(1-\frac{\gamma_{1}}{2}\right) E\left(x_{3}, \infty\right), \quad x_{3} \geq 0 .
$$

Moreover, inequality (5.29), which remains valid under the present conditions, implies

$$
-H(0) \leq\left[1+\frac{1}{2}\left(\frac{\widetilde{\beta}^{2}}{c_{0} \Lambda \lambda_{1} k_{1}}\right)^{1 / 2}\right] E(0, \infty)
$$

and in consequence, the bound (5.34) is recovered.

\subsection{Uniqueness}

Uniqueness of the displacement and temperature is immediate from the last Proposition.

Corollary 5.2 For the Dirichlet boundary value problem defined on the semiinfinite prismatic cylinder $\Omega$, within the class of displacements and temperature for which the total energy is bounded, there is at most only one solution.

Remark 5.1 Other boundary conditions may be similarly treated, although for Neumann boundary conditions, uniqueness holds only to within rigid body displacements and constant temperatures.

Proof: Without loss, the end base displacement and temperature may be assumed to vanish so that from the definition, we have that $H(0)=0$, and consequently (5.31) implies that $H\left(x_{3}\right)=0, x_{3} \geq 0$. We then have from (5.11) that $E(0, \infty)=0$ and the conclusion follows by standard arguments.

\subsection{Decay estimate in terms of base data}

Decay estimates for the solution measured either by the cross-section energy flux or by energy stored in a partial volume are established in Section 5.3. However, the practical utility of these estimates is complete only when the amplitude functions, either $H(0)$ or $E(0, \infty)$, are bounded in terms of base data. We derive such a bound for $E(0, \infty)$ subject to Dirichlet base data, and for this 
purpose recall the continuously differentiable functions $v_{1}(x), \phi(x)$ introduced in Section 4.4 and defined to possess the properties (4.22)-(4.24). Let

$$
\begin{aligned}
e_{i j}(v)= & \frac{1}{2}\left(v_{i, j}+v_{j, i}\right), \\
E^{(v)}\left(y_{3}, x_{3}\right)= & \int_{\Omega\left(y_{3}, x_{3}\right)} c_{i j k l} e_{i j}(v) e_{k l}(v) d x \\
& +\Lambda \int_{\Omega\left(y_{3}, x_{3}\right)} k_{i j} \phi_{, i} \phi_{, j} d x
\end{aligned}
$$

where $\Lambda$ satisfies the condition inherent in (5.8).

On supposing that all source terms vanish, we multiply $(2.25)$ by $v_{i}$, add to (2.11) multiplied by $\Lambda \phi$, integrate by parts over $\Omega$, and note the upper bound (5.13) to obtain

$$
\begin{aligned}
\left(1-\frac{\gamma_{1}}{2}\right) E(0, \infty) \leq & \int_{\Omega}\left(c_{i j k l} e_{i j}(v) e_{k l}+\beta_{i j} e_{i j}(v) \theta+\Lambda k_{i j} \theta_{, i} \phi_{, j}\right) d x \\
\leq & \frac{\gamma_{4}}{2} \int_{\Omega} c_{i j k l} e_{i j} e_{k l} d x \\
+ & {\left[\left(\frac{\widetilde{\beta}^{2}}{c_{0} \lambda_{1} k_{1}}\right)^{1 / 2} \frac{\gamma_{5}}{2}+\frac{\Lambda \gamma_{6}}{2}\right] \int_{\Omega} k_{i j} \theta_{, i} \theta_{, j} d x } \\
& +\left[\frac{1}{2 \gamma_{5}}\left(\frac{\widetilde{\beta}^{2}}{c_{0} \lambda_{1} k_{1}}\right)^{1 / 2}+\frac{1}{2 \gamma_{4}}\right] \int_{\Omega} c_{i j k l} e_{i j}(v) e_{k l}(v) d x \\
& +\frac{\Lambda}{2 \gamma_{6}} \int_{\Omega} k_{i j} \phi_{, i} \phi_{, j} d x
\end{aligned}
$$

where the Schwarz, Poincaré, and arithmetic-mean inequalities are employed, and $\gamma_{4}, \gamma_{5}, \gamma_{6}$ are arbitrary positive constants chosen to satisfy

$$
\begin{aligned}
\gamma_{4} & =\left(1-\frac{\gamma_{1}}{2}\right) \\
\gamma_{5} & =\gamma_{6} \\
& =\Lambda\left(1-\frac{\gamma_{1}}{2}\right)\left[\Lambda+\left(\frac{\widetilde{\beta}^{2}}{c_{0} \lambda_{1} k_{1}}\right)^{1 / 2}\right]^{-1} .
\end{aligned}
$$

In consequence, inequality (5.35) yields

$$
\left(1-\frac{\gamma_{1}}{2}\right) E(0, \infty) \leq c_{11} \int_{\Omega} c_{i j k l} e_{i j}(v) e_{k l}(v) d x+\Lambda c_{12} \int_{\Omega} k_{i j} \phi_{, i} \phi_{, j} d x,
$$

where the constants $c_{11}, c_{12}$ are given by

$$
\begin{aligned}
& c_{11}=\left[\frac{1}{\gamma_{4}}+\frac{1}{\gamma_{5}}\left(\frac{\widetilde{\beta}^{2}}{c_{0} \lambda_{1} k_{1}}\right)^{1 / 2}\right], \\
& c_{12}=\frac{1}{\gamma_{5}},
\end{aligned}
$$


and $\gamma_{4}, \gamma_{5}$ are given by (5.36) and (5.37).

Upon selecting the functions $v_{i}$ and $\phi$ to be given by (4.26) and (4.27), the upper bound becomes

$$
\left(1-\frac{\gamma_{1}}{2}\right) E(0, \infty) \leq \frac{1}{2 s} D_{9}-D_{10}+\frac{s}{2} D_{11},
$$

in which

$$
\begin{aligned}
D_{9} & =\left(c_{11} D_{3}+\Lambda c_{12} D_{6}\right), \\
D_{10} & =\left(c_{11} D_{4}+\Lambda c_{12} D_{7}\right), \\
D_{11} & =\left(c_{11} D_{5}+\Lambda c_{12} D_{8}\right),
\end{aligned}
$$

and

$$
\begin{aligned}
& D_{3}=\int_{D(0)} c_{i \alpha j \beta} w_{i, \alpha} w_{j, \beta} d S, \\
& D_{4}=\int_{D(0)} c_{i \alpha j 3} w_{i, \alpha} w_{j} d S, \\
& D_{5}=\int_{D(0)} c_{i 3 j 3} w_{i} w_{j} d S, \\
& D_{6}=\int_{D(0)} k_{\alpha \beta} \chi_{, \alpha} \chi, \beta \\
&=\int_{D(0)} k_{\alpha 3} \chi, \alpha \\
& D_{7} \chi d S, \\
& D_{8}=\int_{D(0)} k_{33} \chi^{2} d S .
\end{aligned}
$$

Optimisation with respect to $s$ produces a final upper bound represented by

$$
\left(1-\frac{\gamma_{1}}{2}\right) E(0, \infty) \leq\left[\sqrt{\left(D_{9} D_{11}\right)}-D_{10}\right],
$$

which implies and improves the bound (4.28) determined by similar, but less exact, arguments in Section 4.4. Standard inequalities demonstrate that the expression on the right of (5.38) is non-negative. Indeed, because $w_{i}\left(x_{\alpha}\right), \chi\left(x_{\alpha}\right)$ 
are independent of $x_{3}$, we have

$$
\begin{aligned}
D_{4}= & \int_{D(0)} c_{i j k l} w_{i, j} w_{k} n_{l} d S \\
= & \frac{1}{4} \int_{D(0)} c_{i j k l}\left(w_{i, j}+w_{j, i}\right)\left(w_{k} n_{l}+w_{l} n_{k}\right) d S \\
\leq & \frac{1}{4}\left[\int_{D(0)} c_{i j k l}\left(w_{i, j}+w_{j, i}\right)\left(w_{k, l}+w_{l, k}\right) d S\right]^{1 / 2} \\
& \times\left[\int_{D(0)} c_{i j k l}\left(w_{i} n_{j}+w_{j} n_{i}\right)\left(w_{k} n_{l}+w_{l} n_{k}\right) d S\right]^{1 / 2} \\
= & {\left[\int_{D(0)} c_{i j k l} w_{i, j} w_{k, l} d S \int_{D(0)} c_{i j k l} w_{i} n_{j} w_{k} n_{l} d S\right]^{1 / 2} } \\
= & {\left[\int_{D(0)} c_{i \alpha k \beta} w_{i, \alpha} w_{k, \beta} d S \int_{D(0)} c_{i 3 k 3} w_{i} w_{k} d S\right]^{1 / 2} } \\
= & D_{3}^{1 / 2} D_{5}^{1 / 2} .
\end{aligned}
$$

Similarly, we have

$$
D_{7} \leq D_{6}^{1 / 2} D_{8}^{1 / 2} .
$$

But by the arithmetic-geometric mean inequality, it follows that

$$
2\left(D_{3} D_{5} D_{6} D_{8}\right)^{1 / 2} \leq\left(D_{5} D_{6}+D_{3} D_{8}\right),
$$

and consequently

$$
\left(c_{11} D_{4}+\Lambda c_{12} D_{7}\right)^{2} \leq\left(c_{11} D_{3}+\Lambda c_{12} D_{6}\right)\left(c_{11} D_{5}+\Lambda c_{12} D_{8}\right),
$$

which implies that the right side of (5.38) is non-negative.

\section{Further comment}

The method outlined in the previous section may be applied to unbounded bodies contained between parallel planes for which Poincaré's inequality remains valid in the form used. For other types of unbounded bodies, Poincaré's inequality is replaced by Wirtinger's inequality in the construction of a differential inequality with respect to measures taken over spherical or other suitable curvilinear surfaces. An application to isothermal elasticity presented in [13] demonstrates that decay is characteristically algebraic and not exponential, but whether such behaviour generally occurs in corresponding thermoelastic problems awaits clarification. Exact solutions, however, derived in [15] to certain problems in isotropic thermoelasticity confirm that for these particular problems the decay is algebraic. 


\section{References}

[1] K.A.Ames and B.Straughan (1997). Non-standard and Improperly Posed Problems. San Diego London New York: Academic Press.

[2] D'Apice, C., Ciarletta, M. and Chiriţă,S. (2011). Saint-Venant decay rates for an inhomogeneous isotropic linear thermoelastic strip. J.Math.Anal.Appl., 381,121-133.

[3] G.Fichera (1997). Private communication.

[4] J.N.Flavin, R.J.Knops and L.E.Payne (1989). Decay estimates for the constrained elastic cylinder of variable cross-section. Q.Appl. Math., 47, 325350 .

[5] M.E.Gurtin (1972). The Linear Theory of Elasticity. Handbuch der Physik (Ed. S.Flügge) VIa/2, 1-295. Berlin: Springer-Verlag.

[6] D.Iesan. (1980). Incremental equations in thermoelasticity. J. Thermal Stresses, 3, 41-56.

[7] C.O.Horgan (1989). Recent developments concerning Saint-Venant's principle: an update. Appl. Mech.Rev., 42, 295-303.

[8] C.O.Horgan (1996). Recent developments concerning Saint-Venant's principle: a second update. Appl. Mech.Rev., 49, S101-S111.

[9] C.O.Horgan and J.K.Knowles (1983). Recent developments concerning Saint-Venant's principle. In: Advances in Applied Mechanics (Eds.:J.W.Hutchinson and T.Y. Wu) 23, 179-269. New York London: Academic Press.

[10] R.J.Knops (2001). Elements of Elastic Stability Theory. In: Topics in Finite Elasticity. CISM Courses and Lectures 424 (Eds.: Michael Hayes and Giuseppe Saccomandi). Wien New York: Springer-Verlag.

[11] R.J.Knops and L.E.Payne (1971). Uniqueness Theorems in Linear Elasticity. Berlin Heidelberg New York: Springer-Verlag.

[12] R.J.Knops and L.E.Payne (1996). The effect of a variation in the elastic moduli on Saint-Venant's principle for a half-cylinder. J.Elast., 44, 161182.

[13] R.J.Knops, L.E.Payne, and S.Rionero. (1990). Saint-Venant's principle on unbounded domains. Proc. Roy. Soc. Edin., 115A,319-336.

[14] R.J.Knops and R.Quintanilla (2010). Introductory Topics in the Mathematical Theory of Continuum Mechanics. Unesco Encylopedia of Life Support Systems. (Eds.:J. Merodio and G. Saccomandi) Chpt. 6.161.20. Springer On-line. 
[15] R.J.Knops and R.Quintanilla (2013). Spatial and structural stability in thermoelastostatics. In: Encyclopedia of Thermal Stresses (ed: R. Hetnarski). Chpt. 787. DOI 10.1007/978-94-007-2739-7. Dordrecht: Springer-Verlag.

[16] R.J.Knops and E.W.Wilkes (1973). Theory of Elastic Stability. Handbuch der Physik (Ed.: C. Truesdell) VIa/3,125-302. Berlin Heidelberg New York: Springer-Verlag.

[17] A.E.H.Love (1952). A Treatise on the Mathematical Theory of Elasticity. Fourth Edition. Cambridge: Cambridge University Press.

[18] C.Lupoli (1993). A Phragmén-Lindelöf principle for the thermoelastic cylinder of variable cross-section. Meccanica 28, 315-325.

[19] L.E.Payne (1975). Improperly Posed Problems in Partial Differential Equations. Regional Conference Series in Applied Mathematics 24. Philadelphia: SIAM.

[20] N. Weck, (1976). An explicit Saint-Venant's principle in three-dimensional elasticity. In: Ordinary and Partial Differential Equations (Eds.: W N Everitt and B Sleeman) Dundee (1976). Lecture Notes in Mathematics, 564, 518-526. Berlin. Springer-Verlag. 